Revue européenne des sciences sociales

European Journal of Social Sciences

XL-124 | 2002

Histoire, philosophie et sociologie des sciences

\title{
Histoire, philosophie et sociologie des sciences
}

\section{Giovanni Busino}

\section{OpenEdition}

\section{Journals}

Édition électronique

URL : http://journals.openedition.org/ress/596

DOI : $10.4000 /$ ress.596

ISSN : 1663-4446

\section{Éditeur}

Librairie Droz

\section{Édition imprimée}

Date de publication : 1 août 2002

Pagination : 309-317

ISBN : 2-600-00806-3

ISSN : 0048-8046

Référence électronique

Giovanni Busino, « Histoire, philosophie et sociologie des sciences », Revue européenne des sciences sociales [En ligne], XL-124 | 2002, mis en ligne le 01 décembre 2009, consulté le 19 avril 2019. URL http://journals.openedition.org/ress/596; DOI : 10.4000/ress.596 


\section{Giovanni BUSINO}

\section{HISTOIRE, PHILOSOPHIE ET SOCIOLOGIE DES SCIENCES}

La richesse et l'originalité des communications réunies dans ce volume lancent un défi impossible à celui qui prétendrait en ébaucher une synthèse. Un compte rendu des discussions que ces communications suscitèrent est également irréalisable. Pour saisir l'ardeur des débats, pour décrire les états d'esprit, les émotions, les rapprochements, les heurts, les alternances de passion et d'indifférence, les oppositions et les convergences, il aurait fallu établir un récit historique, esquisser une analyse sociologique, ce qui aurait exigé du temps, de la distance et du talent.

Étant donné que l'organisateur du colloque en est totalement dépourvu, il se limitera en conséquence à porter un regard fugace sur les traits majeurs dégagés par les communications et les débats dans les tendances actuelles de la recherche. Il le fera en énumérant quatre séries de remarques qui sont le produit d'une lecture personnelle et expéditive de contributions ici réunies. Par ailleurs, celles-ci se prêtent bien à cet exercice puisqu'elles sont assez diverses pour susciter des interrogations et suffisamment ouvertes pour rendre celles-ci possibles.

Les travaux ici réunis peuvent être regroupés autour de quatre thèmes principaux: 1. La connaissance; 2. Le réalisme et le constructivisme; 3. L'objectivité; 4. La crise et/ou la recomposition des études sur les sciences.

Le lecteur indulgent et les auteurs des textes pardonneront, on l'espère, la manière, télégraphique et assertorique, de traiter ces quatre points.

\section{LA CONNAISSANCE}

Nous disions jusqu'ici qu'elle était constituée par les actions non du sujet psychologique individuel mais du sujet épistémique sur le réel. Ce sujet épistémique dont les coordinations générales des actions dérivent de l'universel de l'organisation biologique, représente, selon la terminologie de Jean Piaget, tout ce qu'il y a de commun à tous les sujets d'un même niveau de développement. Les cognitivistes (matérialistes, éliminationnistes, physicalistes, fonctionnalistes), à leur tour, ont ancré la connaissance dans les neurones et dans la phylogenèse, c'est-àdire dans le biologique, ou la ramènent aux modèles du traitement intelligent de l'information, c'est-à-dire à l'approche computationnelle, et font ainsi de l'intelligence artificielle le modèle de l'intelligibilité de la connaissance.

Pour connaître, ce sujet universel ou épistémique doit construire ou reconstruire l'objet de la connaissance, saisir les mécanismes de cette construction, les produire en pensée, reconstituer le mode de production d'un phénomène, d'un 
événement, d'une action avec des catégories qui structurent ce qu'il y a de commun à tous les sujets.

Les recherches sur ces catégories qui structurent les pensées, qui modèlent les conceptions de l'argumentation et de la preuve, qui organisent les pratiques scientifiques, qui certifient les formes d'explication et qui dotent chacune de ces activités d'une signification symbolique et d'une valeur affective, ces très nombreuses recherches n'ont pas résolu tous les problèmes encore ouverts. Mieux, elles en ont soulevé d'autres. Par exemple, peut-on se passer du sujet psychologique qui est à la fois l'agent et le destinataire de l'intelligibilité de la connaissance? L'analyse du sens et des représentations a mis en évidence les différences entre la connaissance sociale, les connaissances dites ordinaires (pratiques, procédurales, déclaratives, de sens commun, d'arrière plan) et la connaissance scientifique, les normes et les critères permettant la séparation de ces deux formes cognitives et le rapport au sens que la connaissance a pour le sujet.

$\mathrm{Au}$ cours de ces dernières décennies, Bloor, Barnes et puis - dans une perspective plus radicale - Latour ont abordé ces problèmes en revendiquant tour à tour une théorie de l'action scientifique, en élaborant une critique de l'orthodoxie scientiste, en établissant des inventaires des multiples manifestations des objets des sciences et en répétant que la rationalité et l'objectivité sont des notions équivoques. Ils ne sont pas parvenus à libérer ces problèmes de la circularité logique qui les affecte, ni même à distinguer les moyens d'une connaissance scientifique de son objet ou encore à élaborer des modèles permettant de surmonter les difficultés. Cependant, ils ont lancé un défi embarrassant à la fois aux pratiques de recherche et aux manières de les formaliser et de les communiquer.

Pour relever ce défi, faut-il naturaliser toutes les sciences, puisque toutes les opérations cognitives humaines reposent, au final, sur des mécanismes biologiques et évolutionnaires? Ou bien présumer qu'il n'y a aucune différence entre les connaissances ordinaires et la connaissance scientifique mais seulement des écarts causés par l'extension du domaine connu, par les particularités des procédés et des modes de confirmation, par les degrés d'affranchissement des déterminations naturelles et sociales, par l'ajustement de l'intensité des contraintes normatives?

Si la preuve est la seule pierre de touche de la connaissance objective et rationnelle, si l'intelligibilité dérive des critères épistémologiques de l'explication, alors pour valider les expériences sociales, pour éradiquer les préférences individuelles, il faut passer obligatoirement par les catégorisations, les typifications, les taxinomies, par le langage public. Dans ce cas, il est inévitable de couvrir les relations du sujet à la connaissance, ses croyances, ses convictions, ses intuitions, en bref les dimensions subjectives, à la fois cognitives et vécues, de sa participation à la construction de la connaissance, de la compréhension, de l'intelligibilité.

Rendre compte de la complexité des activités cognitives, sans substituer à la question de la vérité celle du dire vrai, à la souveraineté de la raison universelle la discontinuité des figures de la rationalité, oblige à présumer que la rationalité a partie liée avec la croyance, l'intuition, la conviction, à donner une place, un statut, au sujet connaissant.

La communication de Chevalley reformule autrement cette problématique et ouvre des perspectives nouvelles à la théorie du sujet, à la connaissance en tant que processus de fabrication du soi, en tant que technique pour nous constituer en 
sujets de nos actes, pour nous ré-enseigner la puissance d'agir, de sentir, de promettre. Le sujet que la sociologie récente des sciences définit de façon formelle et abstraite débouche sur une connaissance où il n'y a que des controverses, coups bas, compromis abjects, compétitions pour les financements et pour les honneurs. Un sujet s'inventant lui-même, une connaissance comme processus de fabrication du soi, feraient de la communauté scientifique un lieu où des individus acceptent les mêmes modes perceptifs, les mêmes formalismes, les mêmes grandes stratégies interprétatives et peuvent ainsi communiquer leurs expériences dans un langage non amphibologique. C'est l'accord intersubjectif entre des individus à établir la connaissance objective.

La communication de Jacob apporte d'autres matériaux utilisables pour repenser la théorie du sujet. Après avoir défini l'intentionnalité (croyances, jugements) comme «une représentation mentale d'un état de choses ayant conjointement un contenu conceptuel et une condition de vérité», Jacob affirme qu' «une pensée possède un contenu conceptuel complexe qui dépend du contenu des concepts dont elle est formée et de sa structure logique» et qu'il y a des représentations mentales et non mentales. Est-ce que l'intentionnalité, la pensée conceptuelle, la possession des concepts trouvent leur source dans l'appartenance à une communauté? Sont-elles des activités ou des processus sociaux? La réponse, après une analyse fort subtile des deux thèses (l'intentionnalité est intrinsèquement normative, l'intentionnalité est intrinsèquement sociale), est nette: «..., je répudie l'affirmation selon laquelle l'intentionnalité réside dans le pouvoir de représenter ce qui doit être - le devoir être - par opposition à ce qui est. La sémanticité n'est pas intrinsèquement normative. »

Ces recherches, ainsi que les travaux de Pierre Livet sur les émotions et sur les passions, nous obligent à repenser l'activité du sujet, la réversibilité de ses actions et de ses intuitions ainsi que les mécanismes opératoires indispensables à la construction de la connaissance.

\section{RÉALISME ET CONSTRUCTIVISME}

Pour un sociologue, il s'agit de la question la plus difficile à traiter. Le rapport de Kremer-Marietti, véritable état de la question, donne l'occasion de mieux comprendre un certain nombre de questions très compliquées mais aussi de réfléchir autour ou à la marge des problèmes jusqu'ici restés sans solutions.

La notion de réalisme est polyvalente et polysémique. En effet, elle renvoie à l'objectivité (attribution à la réalité extérieure des caractères qui sont, au fait, subjectifs), à la réciprocité (point de vue propre comme absolu), à la relativité (considérer un objet comme existant en soi mais, en réalité, dépendant d'autres objets ou de la perspective caractérisant la perception). La sociologie ne s'est jamais intéressée au réalisme nominal ontologique et au réalisme nominal logique. Elle a réservé une attention particulière au réalisme qui incorpore l'objet à des schèmes et qui fait de la connaissance une mise en relation entre l'objet et le sujet. Une telle option conceptuelle a suscité pourtant maints questionnements. Parmi ceux-ci, le principal peut être formulé ainsi: si l'objet n'existe que dans ses relations avec le sujet, s'il n'est pas une donnée mais le résultat d'une construction au moyen de savoirs objectivés et de partages institués, le processus, le constituant ne peut être 
en même temps que constructif/réflexif et réflexif/constructif. Dans ce cas, le résultat ultime de ce processus est une composition nouvelle, laquelle transcende le déjà construit, assure la non-contradiction, dépasse les constructions achevées et en comble les lacunes.

Les débats actuels entre les réalistes et les antiréalistes, entre les constructivistes et les anticonstructivistes, fondés sur des critères empiriques et narratifs dépourvus de validité, donnent pour acquis le postulat métaphysique selon lequel la réalité existe en soi ou l'assertion que la réalité est construite. Les implications de ces prises de positions renvoient à la problématique de la connaissance ordinaire et de la connaissance scientifique, aux objets, aux quasi-objets, aux relations hybrides et une multitude d'autres questions compliquant et embrouillant la question déjà si complexe de l'objectivité.

Pierre Bourdieu n'a pas tort d'écrire dans Science de la science et réflexivité (2001) que le réel des sciences sociales est «extérieur et indépendant de la connaissance, mais il est lui-même une construction sociale, un produit des luttes antérieures qui, au moins à ce titre, reste un enjeu de luttes présentes. [...] Il faut donc associer une vision constructiviste de la science et une vision constructiviste de l'objet scientifique: les faits sociaux sont socialement construits et tout agent social, comme le savant, construit tant bien que mal, et vise à imposer, avec plus ou moins de force, sa vision singulière de la réalité, son 'point de vue'» (p. 172).

Faut-il reprendre les objets autrement que comme des cadres naturels de l'action, de l'infrastructure matérielle; faut-il inventer des prérequis pour modifier la théorie de l'action, de l'acteur; considérer, selon la suggestion de Jorland, que le concept de révolution scientifique n'est qu'un canon heuristique pour comprendre la science en train de se faire, qu'un concept historique pour problématiser des questions actuelles? Peut-être. Mais les façons pour rendre ces programmes de recherche opératoires sont loin d'être simples et faciles. Bourdieu a dit et redit (p. 222) «que si, comme n'importe quel autre savant, il [sc.: le sociologue] s'efforce de contribuer à la construction du point de vue sans point de vue qu'est le point de vue de la science, il est, en tant qu'agent social, pris dans l'objet qu'il prend pour objet et qu'à ce titre il a un point de vue qui ne coïncide ni avec celui des autres ni avec le point de vue en survol et en surplomb de spectateur quasidivin qu'il peut atteindre s'il accomplit les exigences du champ».

\section{L'OBJECTIVITÉ}

Le rapport de Feldman révèle à la fois la richesse et la complexité d'un tel sujet. Le concept nous renvoie à la fois à des états d'âme, à des états du monde, à des problèmes ontologiques, épistémologiques, méthodologiques, éthiques. Les différentes acceptions du concept ne se superposent, ne se croisent, ne s'intègrent ni en théorie ni en pratique. La norme du vrai sanctionnée dans une communauté scientifique, l'objectivité conquise grâce aux découvertes et moyennant les démonstrations présentes, ne suffisent à épuiser tous les questionnements à son sujet. L'histoire d'une théorie, d'une technique, la recherche en action, la mise en évidence de leurs fonctions sociales, ne disent rien sur leur validité. La théorie de Darwin sur l'évolution, la classification de Linné ou de Buffon, le probabilisme, sont insaisissables avec le seul recours à leurs conditions sociales. Le monde est 
infiniment plus riche que les modèles avec lesquels nous tentons de le saisir. L'attention au local et au singulier, les structures sociales de l'activité scientifique ne disent rien sur le point qu'une découverte, une démonstration, une taxinomie, fabriquées dans un contexte social donné, peuvent devenir universelles et se généraliser d'un contexte à l'autre. Ni l'internalisme ni l'externalisme ne nous aident vraiment à explorer les formes effectivement prises par la production, l'inscription matérielle, la diffusion et la généralisation de tel ou tel autre savoir.

L'objectivité se construit en fonction des activités du sujet; elle se construit grâce à la coordination des actions et des opérations; elle est un mélange indifférencié d'apports du sujet et de l'objet. La décentration, en nous dégageant des entraves du moi, des intrusions allogènes, des illusions des sens, du langage, des points de vue, des valeurs, etc., aide à situer l'action dans le contexte de relations spatiales et causales indépendantes de l'activité immédiate. Dans cette perspective il n'y a pas de place pour les invariances; les objets sont simultanément affectifs et cognitifs, le social et le rationnel ne sont plus des mondes séparés ou différents.

Ceci étant, comment discriminer les diverses natures des croyances, des idées, des descriptions, des valeurs, rendre compte de la subjectivité invariante? Peut-on appliquer le principe de symétrie à des contextes et à des situations incomparables et incommensurables? De quelle façon évaluer la pertinence, la cohérence et la plausibilité des argumentations, des schèmes, des singularités, des savoirs singuliers, lorsqu'ils sont fondés sur des logiques non démonstratives, par exemple sur la logique des sentiments? L'évaluation doit prendre en charge uniquement ce qui est «présent» et négliger ce qui est «absent» car non évident?

Peut-être que nous devrions voir de plus près les thèses développées par Amartya Sen dans Objectivity and Position (1992), approfondir sa distinction entre vérité et objectivité, analyser attentivement ses approches du subjectivisme et du relativisme. Sa théorie de l'objectivité de positionnement ouvre des perspectives nouvelles sur «l'illusion objective», sur le fait que les actions dépendent d'une position spécifique, que nous agissons sur la base de ce que nous avons de bonnes raisons de croire en prenant en compte les informations disponibles. Cette théorie comme par ailleurs celle de Stephen Toulmin dans Return to Reason (2001) ont le mérite de constater que le monde social est un monde de risques et d'incertitudes mais aussi de démontrer que la raison est indispensable à la vie et qu'elle ne doit pas être confondue avec le rationalisme abstrait.

\section{CRISE ET/OU RECOMPOSITION DES SCIENCES SOCIALES}

Ce point a été longuement analysé et débattu au cours des jours du colloque par de très nombreux chercheurs. Par conséquent, il est aisé d'être très bref.

Nicole Ramognino revendique l'ontologie hétérogène des formes sociales en tant que formes symboliques. Pour cette raison, elle prône l'abandon des polarisations classiques individualisme/holisme, acteur/fait, ainsi que la comparaison historico-sociale des formes de rationalité et de son universalisation «dans la mesure où celles-ci sont toujours en même temps une distribution cognitive des savoirs et une division sociale du travail sémantique». 
De son côté, Alban Bouvier analyse, avec beaucoup de perspicacité, en quoi la connaissance scientifique est différente de la connaissance commune et de quelle façon une épistémologie individualiste se corrèle avec une épistémologie sociale. Dans ce texte, il y a une esquisse d'une théorie qui voudrait rendre compte des processus grâce auxquels des sujets multiples prolongent l'analyse individuelle en analyse infra-individuelle.

Fassa discute avec élégance et pondération les travaux les plus récents de Bruno Latour et conclut qu'ils subtilisent aux humains le pouvoir d'effectuer des choix, de maîtriser le monde, de reconnaître que tous les conflits ne peuvent pas aboutir à des compromis, donner lieu à des solutions.

Coenen-Huther, avec la maestria que tout le monde lui reconnaît, trace un panorama suggestif de l'état actuel de la sociologie de la science. Pour lui la science est le produit d'une confrontation disciplinée de point de vue. Les dispositions individuelles du chercheur lui paraissent «d'importance marginale». Il constate un glissement périlleux du social en tant que construction vers le social en tant que représentation. Il souhaite que les sociologues en prennent conscience et en examinent les conséquences pour le développement de leur discipline.

Ragouet, après avoir montré les défaillances des recherches actuelles, plaide pour une sociologie configurationnelle des sciences, pour la construction de configurations de façon inductive, et argumente en faveur d'une universalité pratique et communicationnelle.

Dubois trace les grandes lignes d'un programme de recherche non réductionniste, basé sur une conception forte de l'acteur social, sur un rationalisme élargi non causaliste, sur une réinterprétation et refondation du principe de symétrie. Ce programme, d'inspiration actionniste, privilégie les raisons des acteurs opérant des choix, en prenant des décisions, en se ralliant à une opinion.

Berthelot prend ses distances d'avec les débats entre internalisme et externalisme, entre rationalisme et relativisme, entre réalisme et constructivisme. Après avoir admis que l'activité scientifique est sociale, il ajoute qu'elle est soumise à des règles de cohérence et de pertinence, d'où découle sa spécificité: révision argumentative permanente, dispositifs de mise à l'épreuve et de constructions contrôlées ou contrôlables, révisables ou changeables. Ce programme non réductionniste est étayé grâce à l'analyse d'une controverse célèbre et classique, analyse dont on doit admirer l'élégance, la finesse interprétative et l'érudition.

La lecture des textes réunis ici montre que les sciences sociales ont de la difficulté à admettre les interdépendances des registres historiques, philosophiques, épistémologiques et sociologiques parce que ces disciplines ont un fort penchant pour les praxéologies, une tendance à croire qu'elles sont en mesure d'apporter les bonnes solutions aux problèmes de l'organisation sociale. C'est compréhensible et explicable. En effet, afin de connaître, comprendre et interpréter les phénomènes, naturels ou sociaux, afin d'en dévoiler les structures profondes, les hommes ont si fortement misé sur les apports de la recherche scientifique et des innovations technologiques que ces dernières sont devenues essentielles au fonctionnement et à la survie de la société. Or celles-ci, pour être sûres d'obtenir une production stable et continue, ont dû créer, dès le XVII siècle, des institutions ad hoc. Elles ont dû les doter de pouvoirs et de privilèges importants ainsi que de 
moyens matériels (laboratoires et appareils) et culturels (bibliothèques, congrès, revues, etc.) de plus en plus considérables.

Bien que le rôle de la science et des techniques dans la production des connaissances, dans le développement économique et social, dans l'amélioration des conditions de vie des populations soit incontestable; bien que la capacité de la science à ne jamais s'incliner devant les mystères du réel et à tout soumettre à l'épreuve de l'expérience entièrement prouvée, les critiques contre sa prétention à être une valeur absolue et à constituer la référence ultime pour tous les systèmes normatifs se sont multipliées et radicalisées de façon exponentielle. Pour maîtriser rationnellement tous les phénomènes, naturels et sociaux sont nécessaires l'aide du calcul, de la formalisation et de l'informatique. Cette croyance constituerait, avec l'économie, l'idéologie de la Modernité. Son but serait d'assurer la prééminence hégémonique d'une forme d'organisation sociale sur toutes les autres de la Planète.

La critique de la science, sa réduction à une croyance pareille à n'importe quelle autre croyance sociale, ont mis en péril un certain nombre de préconstruits culturels et surtout les notions d'objectivité et de vérité. Ce réductionnisme a aussi alimenté un relativisme généralisé selon lequel il y a autant de vérités, de valeurs et de points de vue que de groupes sociaux ou de communautés d'intérêts, de cultures diverses, de conceptions du monde. Les universaux de la science ne seraient que les cache-misère de l'idéologie de la Modernité.

Dans les débats actuels sur la science, Bruno Latour (Le Monde, 29.08.1996) a le mérite de synthétiser une telle position avec des formules lapidaires: «Les sciences, les techniques, les marchés, n'ont jamais eu l'aspect lisse, objectif, progressif, inhumain, que les Européens ont souhaité lui donner afin de construire leur idée de progrès. [...] Derrière nous peut-être, dans le passé, nous confondions les faits et les valeurs, les sciences et les politiques, mais devant nous, à coup sûr, le nœud qui relie les faits, ce que sont les choses et les valeurs, ce que veulent les humains, se trouvera plus serré encore, plus indémêlable [...]. Nous n'avons jamais avancé vers un surcroît d'efficacité et de rentabilité...». D'après ce chercheur, le divorce entre la philosophie des sciences et la sociologie, entre l'épistémologie et l'histoire sociale des sciences, a été provoqué par une conception réduisant le social aux seuls humains, à leurs seuls rapports sociaux, à leurs seuls intérêts économiques. «Le social se compose, en large partie, de non-humains mobilisés en son sein [...]. Il produit [...] des nouvelles associations, des combinaisons inouïes d'humains et non-humains [...]. Une société ne se compose pas de sujet de droit en retrait par rapport aux choses, mais de multiples associations et de multiples échanges entre humains et non-humains. »

Que sont ces non-humains? D'où viennent-ils? De quels droits sont-ils titulaires? Ont-ils les mêmes responsabilités que les humains?

Latour donne aux objets un statut d'acteur et propose de remplacer l'intersubjectivité par l'interobjectivité, de définir l'interaction sociale en y introduisant les objets. Cependant, il ne nous dit pas comment des objets, construits ou valorisés par des acteurs sociaux, deviennent subitement, eux aussi, des acteurs protagonistes d'interactions sociales. Il y a là un mystère, voir un tour de passe-passe. Peut-on fonder ces thèses sur la réduction de la science à une simple croyance sociale, semblable ou analogue à la magie ou à l'idéologie d'une «illusion», l'illusion de la modernité? Peut-on traiter la science en tant que totalité, globalité, 
alors qu'elle sépare, distingue, morcelle en lots, et procède par approximations successives, par tâtonnements, corrections et rectifications? Comment oublier le fait que les savants s'intéressent avant tout à la validité des conclusions particulières sur le réel, alors que les philosophes s'intéressent à la nature de la méthode scientifique en sa globalité, que les premiers ne prêtent aucune attention à la question des fondements alors que, pour les seconds, elle est primordiale et absolue?

Les connaissances scientifiques passent aussi par des processus intellectuels, par des élaborations conceptuelles, par la critique des savoirs. Est-il raisonnable de négliger ce travail essentiel et de valoriser exclusivement les conflits de pouvoirs, les contraintes institutionnelles, les enjeux médiatiques, les conditionnements socio-politiques?

Les discours sur les fondements nous portent à quêter l'absolu, alors que nous devons nous contenter de réponses vérifiables bien que provisoires. La science est une croyance spécifique, à la croisée de dépendances et d'interdépendances spécifiques avec le contexte global. Traiter la science et la technique comme si elles étaient des croyances constitutives de la modernité nous conduit à une impasse intellectuelle. La science et la technique sont vides de finalités, elles ne sont que des moyens au service des sociétés et celles-ci les utilisent pour le meilleur et pour le pire. Attribuer à la science et à la technique les méfaits du colonialisme, les abus du capitalisme et du socialisme, les totalitarismes de droite et de gauche, le développement incontrôlé, la pauvreté, le paupérisme, le chômage, l'effet de serre, la mise en danger de l'écosystème, les difficultés existentielles auxquelles nous sommes actuellement confrontés, la perte des liens sociaux, l'exclusion, la marginalisation et tant d'autres maux dont nos sociétés souffrent, c'est une vision simpliste et dangereuse. Simpliste parce qu'elle ne propose qu'une seule façon de voir et de concevoir un ensemble de choses complexes; dangereuse parce qu'elle nous replonge dans le mysticisme et dans l'irrationalisme, elle nous livre aux bizarreries des prophètes et aux pratiques intellectuelles et sociales régressives et irresponsables. Par contre, si nous voulons déchiffrer les énigmes de la vie sociale et décoder les mystères du monde naturel, si nous voulons maîtriser les événements et en contrôler les développements, le seul moyen dont nous disposons est la méthode scientifique. Grâce à cette méthode, à l'aide de données empiriques susceptibles de vérifier nos hypothèses et nos formulations théoriques, la science peut nous faire comprendre le monde dans lequel nous nous trouvons et agissons.

Nous savons depuis David Hume que l'induction nous enferme dans un cercle vicieux (soit à justifier une généralisation au moyen d'une autre généralisation légitimée par son succès passé) et qu'il est impossible de fonder logiquement la science. Karl Poppper est allé plus loin dans cette même direction grâce à son principe de falsification, dont l'observation sociologique du travail dans les laboratoires a toutefois montré les limites. En effet, le travail réel des savants consiste surtout à comprendre pourquoi les hypothèses adoptées ne fonctionnent pas, plutôt qu'à en chercher la réfutation ou à en démontrer la fausseté. En outre, dans les relations causales du monde réel, il n'est pas donné d'observer un rapport d'une seule cause à un seul effet. Les phénomènes réels sont «causés » par de très nombreuses variables, ils se trouvent en situation de mutuelle dépendance. L'histoire des pratiques scientifiques nous prouve que la science ne constitue pas un corpus fermé de théories. Elle n'est qu'un ensemble d'instructions, de préceptes et de prescriptions servant à faire des classifications, des inférences causales, à formu- 
ler des hypothèses, à vérifier, à organiser les observations en séquences logiques et cohérentes, à identifier les différents niveaux d'explications, les disparités technologiques et les diverses capacités cognitives. Les résultats produits sont universaux pour tous ceux qui appliquent, avec un esprit critique, ces modèles normatifs. Ces universaux ne sont pas éternels, jamais définitifs. Ils se modifient en fonction des contraintes culturelles, des contextes sociaux et cognitifs, de l'urgence des problèmes à résoudre, des curiosités intellectuelles à satisfaire, mais toujours selon des processus propres à l'activité scientifique, à son autonomie conceptuelle et à son organisation, à la constance et à la permanence des contrôles critiques.

C'est par là que je voudrais terminer. J'espère que nous serons capables d'arrêter la glissade des sciences sociales, et notamment de la sociologie, sur la pente du déclin. J'espère que, dans dix ans, il n'y aura plus personne à répéter ce refrain, qu'on sera en condition de prendre conscience que la sociologie, en collaboration avec la philosophie et l'histoire, contribuera à identifier les nouveaux possibles, qu'elle sera un instrument de libération, une pensée qui médite et réfléchit.

Institut d' anthropologie et de sociologie

Université de Lausanne

\section{RÉFÉRENCES}

P. Bourdieu, Science de la science et réflexivité..., (2001) 172/222.

B. Latour, Le Monde, La modernité est terminée, 29.08.1996, p. 11.

A. Sen, Objectivity and Position..., 1992.

S. Toulmin, Return to Reason..., 2001. 